\title{
Intracranial Hypertension due to Cerebral Venous Sinus Thrombosis following Head Trauma: A Report of Two Cases
}

\author{
Peter Lindvall Lars-Owe D. Koskinen \\ Division of Neurosurgery, Department of Pharmacology and Clinical Neuroscience, \\ Umeå University, Umeå, Sweden
}

\section{Key Words}

Head trauma · Cerebral venous sinus thrombosis · Intracranial hypertension · Thrombectomy · Decompressive craniectomy

\begin{abstract}
Cerebral venous sinus thrombosis (CVST) may occur following head trauma and contribute to intracranial hypertension that mandates immediate action. Anticoagulant therapy is the first line of treatment in CVST but may not be applicable in patients with head trauma. Here, we report on the treatment of 2 patients with CVST. In 1 patient, there was an attempt to perform thrombectomy and thrombolysis, and eventually a decompressive craniectomy was performed. In this patient, there was an excellent outcome. In the other patient, an immediate decompressive craniectomy was performed that did not improve the outcome.
\end{abstract}

C 2013 S. Karger AG, Basel

\section{Introduction}

Cerebral venous sinus thrombosis (CVST) is a serious complication that may occur following head trauma and lead to increased intracranial pressure (ICP), venous infarctions and death $[1,2]$. The prevalence following head trauma is uncertain, but skull fractures near a dural venous sinus or the jugular bulb are a risk factor for developing CVST [3]. The best management of these patients remains unclear because the use of systemic anticoagulation or local thrombolysis may be hazardous for patients with previous head trauma. Good outcomes have been reported following decompressive hemicraniectomy for patients with 
Lindvall et al.: Intracranial Hypertension due to Cerebral Venous Sinus Thrombosis following Head Trauma: A Report of Two Cases

CVST and hemorrhagic infarctions [4, 5]. There are only a few reports on patients with posttraumatic CVST or venous obstruction $[2,6,7]$, and most have reported on patients with a moderately increased ICP or a delay of 2-8 days before developing oedema or venous infarctions. Here, we report on the management of 2 patients with severely increased ICP, mandating immediate action.

\section{Case Reports}

\section{Patient 1}

The patient was a 13-year-old female who was hit by a car while riding a horse. She was described as having a Glasgow coma scale score of 9 and intubated prior to transport to the neurosurgical department at Umeå University Hospital. During clinical examination with a low dose of propofol, the patient showed withdrawal to painful stimuli in all extremities and the pupils were of normal size and responsive to light. A CT scan showed unremarkable findings with only some minimal contusions and otherwise visible sulci on the convexities and open basal cisterns (fig. 1a, b). There was no visible skull fracture. The ICP, measured by an intraparenchymal pressure monitor (Codman MicroSensor; Johnson \& Johnson), however, was $30 \mathrm{~mm} \mathrm{Hg}$. Despite sedation with phenobarbital and the placement of an external ventricular drainage for intermittent drainage of cerebrospinal fluid, the ICP was 30-35 mm Hg. To exclude venous thrombosis, an angiography was performed 2 days later that showed thrombosis in the right transverse sinus (TS) and contrast stagnation in cortical veins, including the vein of Labbé, as a sign of impaired venous drainage (fig. 1c). The right femoral vein was catheterized and a microcatheter (Renegade; Boston Scientific) was inserted into the right TS in an attempt to perform a mechanical thrombectomy. This maneuver, however, did not provide a lasting effect; $20 \mathrm{mg}$ of tissue plasminogen activator was instead administered locally as a bolus. Angiographic imaging showed a partial resolution of the thrombus with flow in the TS (fig. 1d), whereas the ICP increased to $70 \mathrm{~mm}$ Hg. A CT scan showed newly formed subarachnoid haemorrhage, acute subdural haematomas bilaterally and progress of frontal contusions (fig. 1e). Evacuation of acute subdural haematomas and a bifrontal hemicraniectomy were performed immediately, and the postoperative ICP remained stable at $<20 \mathrm{~mm} \mathrm{Hg}$ (fig. 1f). The patient was then treated with dalteparin $(2,500 \mathrm{U})$ daily during 5 additional days in the ICU until she could be extubated. The patient's recovery following a cranioplasty 2 months later was excellent, and there were no neurological deficits.

\section{Patient 2}

This patient was a 20-year-old male with a history of drug abuse. The patient had tried to commit suicide by jumping from the second floor of a building and landed on concrete. He was reported to have a Glasgow coma scale score of 9 on admission to hospital. A CT scan showed multiple non-dislocated fractures in the posterior fossa and cerebral contusions in the right cerebellar hemisphere and bifrontally (fig. $2 \mathrm{a}, \mathrm{b}$ ). The patient was intubated and transferred to the neurosurgical department at Umeå University Hospital. A clinical examination with a low dose of propofol revealed that he showed withdrawal to pain in all extremities and that the pupils were of normal size and reactive to light. An intraparenchymal pressure monitor was inserted (Codman MicroSensor; Johnson \& Johnson) that showed an initial ICP of $50 \mathrm{~mm} \mathrm{Hg}$ that soon increased to $80 \mathrm{~mm} \mathrm{Hg}$. A new CT scan showed only slight progress of contusions compared to the initial investigation. The patient was brought to the operating room, and a large bifrontal hemicraniectomy was performed. There was 
profuse venous bleeding from the bone and the dura during the procedure and there were clear signs of venous congestion of the cortical veins. At the end of the procedure, the ICP was approximately $25 \mathrm{~mm} \mathrm{Hg}$; however, it soon increased to $50 \mathrm{~mm} \mathrm{Hg}$. A new CT scan showed a substantial progress of cerebral oedema and frontal contusions, whereas a CT venography showed a thrombosed sigmoid sinus on the right side, a fractured jugular foramen and a thrombus extending down to the jugular vein (fig. 2c-f). The ICP further increased to $80 \mathrm{~mm} \mathrm{Hg}$ and the patient subsequently died of circulatory arrest.

We received consent from the surviving patient in this case report. For obvious reasons, it was not possible to obtain such consent from the patient who died. There was no study protocol that needed approval from an ethical committee.

\section{Discussion}

CVST is a condition that may cause cerebral oedema, venous infarcts and death mainly through transtentorial herniation [1]. Mortality rates have been reported to be 6-10\% [8]. Head trauma may be a cause of venous obstruction either through CVST or impressed bone fragments occluding a venous sinus $[2,6,7]$. Venous obstruction or CVST may elevate ICP following head trauma and complicate the management of these patients. There are, however, only a few reports on this subject, even if CVST may be more common than previously believed. The presence of skull fractures close to dural sinuses or the jugular bulb is a risk factor, and 1 retrospective study showed that $40.7 \%$ of patients with these fractures had CVST [3]. Angiography or magnetic resonance imaging are regarded as the best diagnostic tools [8], but may be impractical or too time-consuming following head trauma. A CT venography has a diagnostic sensitivity of $95 \%$ and may, as in patient 2 , be a fast way to establish the diagnosis [9]. In patient 2, the finding of CVST was initially overlooked even in the presence of multiple fractures near the transverse and sigmoid sinus and involvement of the jugular foramen. The diagnosis was confirmed postoperatively due to findings of venous congestion during surgery.

As in patient 1, CVST may also be suspected due to an increased ICP without sufficient pathology to explain this, even in the absence of skull fractures. In both patients, we believe that CVST substantially affected the ICP. In patient 2, there were cerebral contusions exerting mass effect, but not to the extent to fully explain the severely raised ICP. In nontraumatic CVST, the first line of treatment is anticoagulants such as intravenous heparin or low molecular weight heparin subcutaneously, which has been shown to reduce the risk of death or dependency compared to placebo [10]. This treatment is considered to be safe and has not been shown to promote or worsen intracerebral haemorrhage in patients with nontraumatic CVST [8]. In both of our patients, this treatment would initially not have been an option due to an immediate need to lower the ICP, and also maybe due to the risk of progression of intracranial bleeding. Anticoagulant therapy is generally avoided in patients with severe head trauma. The actual risk of progression of bleeding following anticoagulant therapy in patients with severe head trauma and traumatic CVST has, however, not been evaluated. In patient 1 , we tried, as reported in some series, to perform both mechanical thrombectomy and thrombolysis $[11,12]$. Thrombectomy or thrombolysis has the potential to provide a faster restitution of venous outflow than anticoagulant therapy. The efficacy and safety of these treatments have, however, not been proven in patients with CVST. The use of tissue plasminogen activator in patient 1 led to a progression of bleeding, which mandated us to perform an acute decompressive craniectomy. Decompressive hemicraniectomy may improve outcome in patients with raised ICP and head trauma in general, but it has also been 
reported as a treatment option in patients with CVST and venous infarctions $[4,6,13,14]$. In patient 1 the outcome was excellent, while in patient 2 there was a substantial increase in size of the frontal hemorrhagic contusions and surrounding oedema. This may in part be due to severe venous congestion. The performed decompressive craniectomy did not improve outcome in this case and may even have worsened the situation. However, due to the severely increased ICP and the need for immediate decompression, there would have been no time for thrombectomy or thrombolysis. As seen above, intracranial hypertension may be an acute concern in patients with traumatic and non-traumatic CVST and seems to be the major cause of death in these patients [1]. Mass lesions such as haemorrhages and cerebral oedema may cause intracranial hypertension and transtentorial herniation. Even if decompressive craniectomy may be beneficial to reduce the ICP and buy the treating physician some valuable time, focus must be directed towards restoring venous outflow to avoid oedema, infarctions or haemorrhages. Anticoagulant therapy has been proven effective in the treatment of CVST, but the role of this treatment in patients with previous head trauma and concomitant severe intracranial hypertension remains unclear.

\section{Conclusions}

CVST may occur following head trauma and contribute to intracranial hypertension. Recognition of this condition is important as it may affect outcome. The treatment still remains unclear, especially as there may be an immediate need to lower the ICP. Mechanical thrombectomy or thrombolysis may provide a faster venous restitution than anticoagulant therapy, even if there is still no evidence for the efficacy and safety of this treatment. A decompressive hemicraniectomy is also a possible treatment option to reduce the ICP.

\section{Disclosure Statement}

We received no funding for this project. There are no conflicts of interest.

\section{References}

1 Canhao P, Ferro JM, Lindgren AG, Bousser MG, Stam J, Barinagarrementeria F: Causes and predictors of death in cerebral venous thrombosis. Stroke 2005;36:1720-1725.

2 Matsushige T, Nakaoka M, Kiya K, Takeda T, Kurisu K: Cerebral sinovenous thrombosis after closed head injury. J Trauma 2009;66:1599-1604.

-3 Delgado Almandoz JE, Kelly HR, Schaefer PW, Lev MH, Gonzalez RG, Romero JM: Prevalence of traumatic dural venous sinus thrombosis in high-risk acute blunt head trauma patients evaluated with multidetector CT venography. Radiology 2010;255:570-577.

4 Rajan Vivakaran TT, Srinivas D, Kulkarni GB, Somanna S: The role of decompressive craniectomy in cerebral venous sinus thrombosis. J Neurosurg 2012;117:738-744.

5 Ferro JM, Crassard I, Coutinho JM, Canhao P, Barinagarrementeria F, Cucchiara B, Derex L, Lichy C, Masjuan J, Massaro A, Matamala G, Poli S, Saadatnia M, Stolz E, Viana-Baptista M, Stam J, Bousser MG: Decompressive surgery in cerebrovenous thrombosis: a multicenter registry and a systematic review of individual patient data. Stroke 2011;42:2825-2831.

6 Dobbs TD, Barber ZE, Squier WL, Green AL: Cerebral venous sinus thrombosis complicating traumatic head injury. J Clin Neurosci 2012;19:1058-1059.

7 Yokota H, Eguchi T, Nobayashi M, Nishioka T, Nishimura F, Nikaido Y: Persistent intracranial hypertension caused by superior sagittal sinus stenosis following depressed skull fracture. Case report and review of the literature. J Neurosurg 2006;104:849-852.

-8 Masuhr F, Mehraein S, Einhaupl K: Cerebral venous and sinus thrombosis. J Neurol 2004;251:11-23. 
Lindvall et al.: Intracranial Hypertension due to Cerebral Venous Sinus Thrombosis following Head Trauma: A Report of Two Cases

-9 Casey SO, Alberico RA, Patel M, Jimenez JM, Ozsvath RR, Maguire WM, Taylor ML: Cerebral CT venography. Radiology 1996;198:163-170.

10 Einhaupl K, Stam J, Bousser MG, De Bruijn SF, Ferro JM, Martinelli I, Masuhr F: EFNS guideline on the treatment of cerebral venous and sinus thrombosis in adult patients. Eur J Neurol 2010;17:1229-1235.

11 Ciccone A, Canhao P, Falcao F, Ferro JM, Sterzi R: Thrombolysis for cerebral vein and dural sinus thrombosis. Cochrane Database Syst Rev 2004;CD003693.

12 Rahman M, Velat GJ, Hoh BL, Mocco J: Direct thrombolysis for cerebral venous sinus thrombosis. Neurosurg Focus 2009;27:E7.

13 Guerra WK, Gaab MR, Dietz H, Mueller JU, Piek J, Fritsch MJ: Surgical decompression for traumatic brain swelling: indications and results. J Neurosurg 1999;90:187-196.

14 Olivecrona M, Rodling-Wahlstrom M, Naredi S, Koskinen LO: Effective ICP reduction by decompressive craniectomy in patients with severe traumatic brain injury treated by an ICP-targeted therapy. J Neurotrauma 2007;24:927-935. 
Lindvall et al.: Intracranial Hypertension due to Cerebral Venous Sinus Thrombosis following Head Trauma: A Report of Two Cases

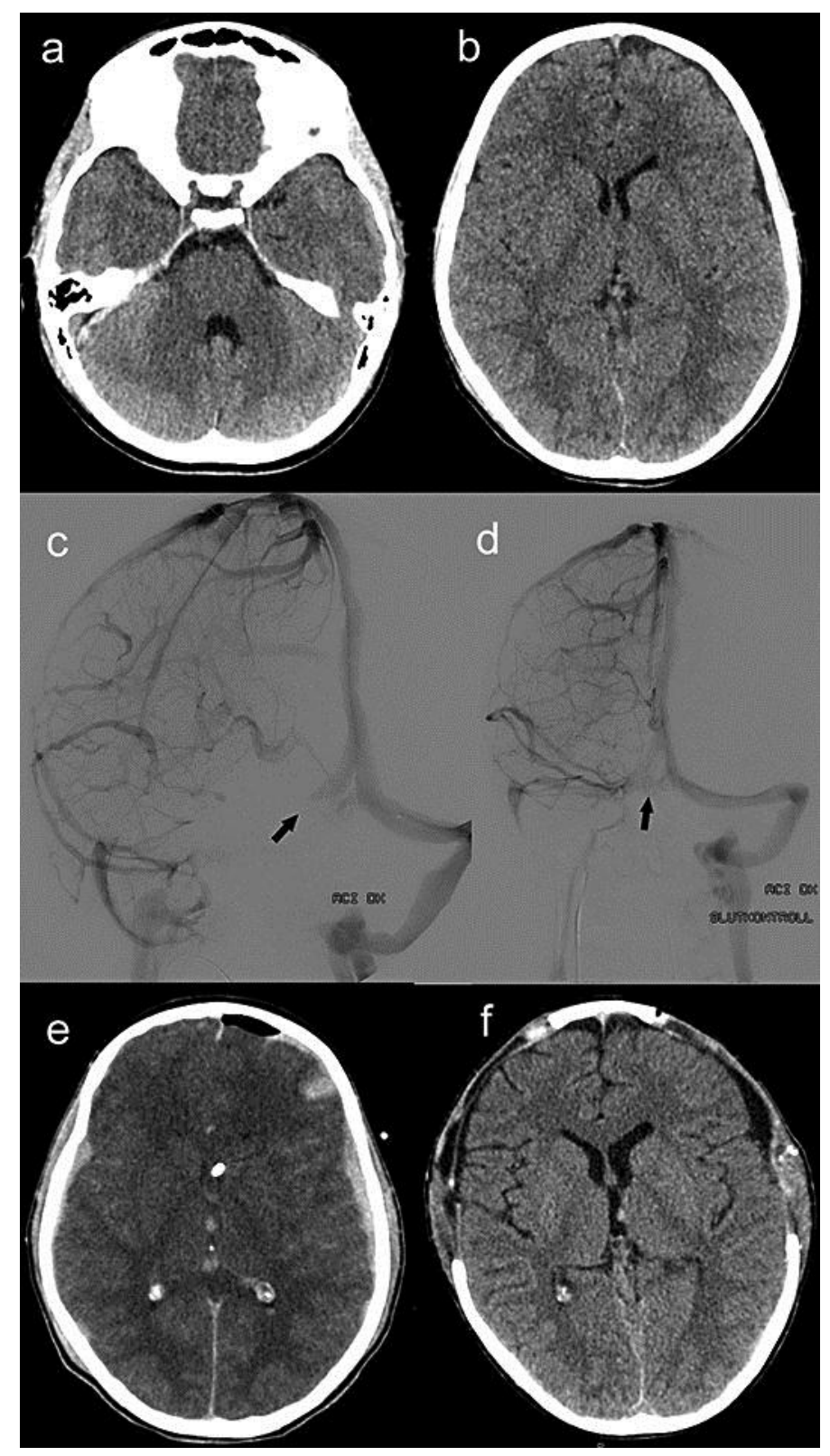

Fig. 1. a, b CT scan following head trauma. c Angiogram shows thrombosis in the TS on the right side (arrow). d Partial recanalisation of the TS (arrow) following $20 \mathrm{mg}$ of tissue plasminogen activator. e CT scan shows progress of a frontal contusion on the left side and new bilateral acute subdural haematomas. f CT scan following bilateral craniectomy. 
Lindvall et al.: Intracranial Hypertension due to Cerebral Venous Sinus Thrombosis following Head Trauma: A Report of Two Cases

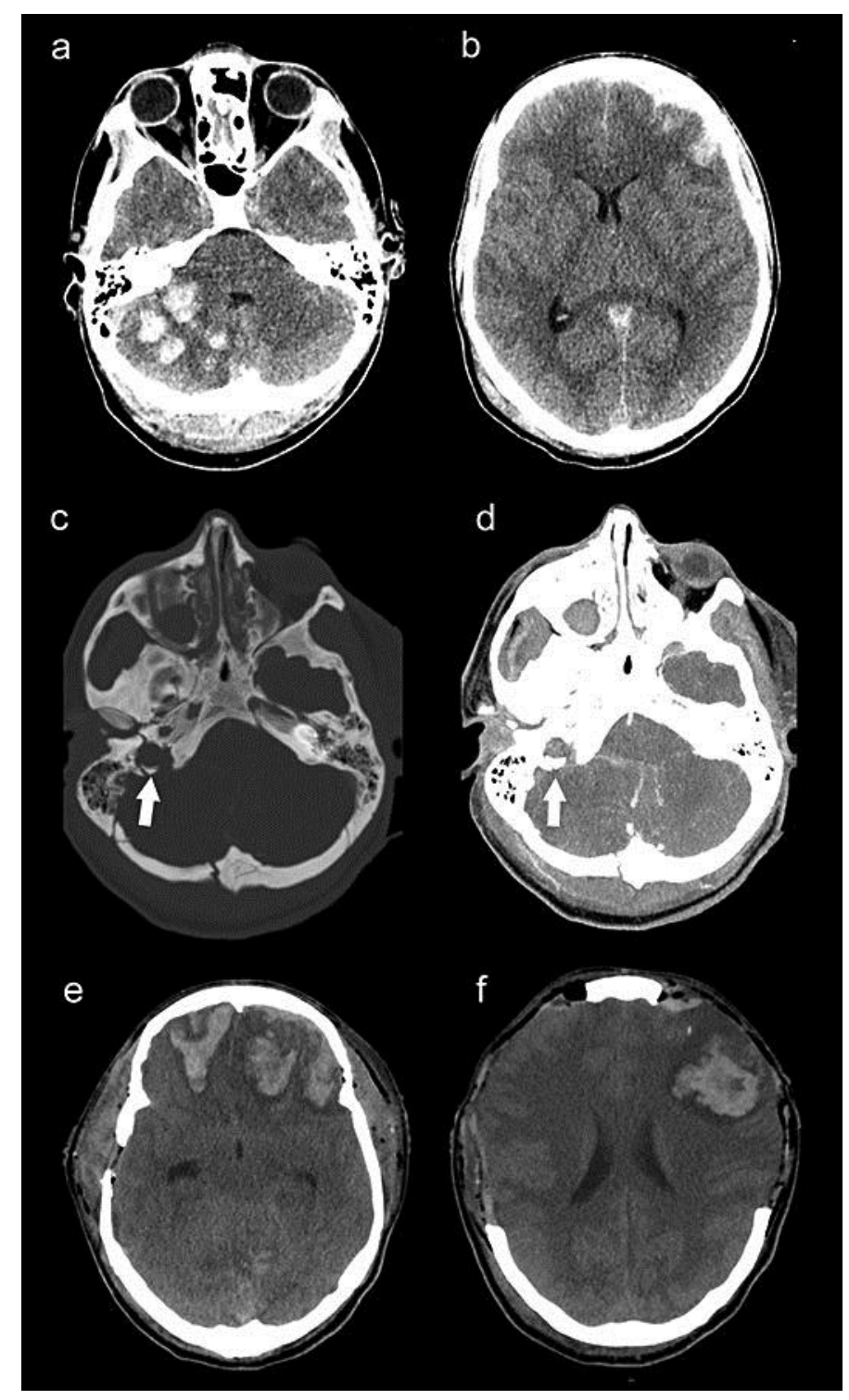

Fig. 2. a, b CT scan shows cerebellar contusions on the right side and a frontal contusion on the left side. c CT scan with a bone window shows fractures in the posterior fossa engaging the jugular foramen (arrow). d CT venography shows venous thrombosis in the level of the jugular foramen (arrow). e, $\mathbf{f}$ CT scan following bilateral craniectomy shows progress of frontal contusions. 